\title{
INFORMES DE RESULTADOS DE 2016 Y 2017 SOBRE LAS RECOMENDACIONES PROPUESTAS POR EL GRECO PARA LA PREVENCIÓN DE LA CORRUPCIÓN JUDICIAL EN ESPAÑA: CRÓNICA DE INCUMPLIMIENTOS
}

\author{
JOSÉ ANGEL CAMISÓN YAGÜE \\ Profesor Contratado Doctor de Derecho Constitucional \\ Universidad de Extremadura
}

SILVIA SORIANO MORENO

Profesora de Derecho Constitucional

Universidad de Extremadura

\section{SUMARIO}

I. Introducción. II. Recomendaciones del GREGO para evitar la corrupción en el Poder Judicial: control de su cumplimiento. III. Conclusiones.

\section{INTRODUCCIÓN}

El presente trabajo tiene por objeto analizar, en aquello específicamente referido al Poder Judicial, los Informes de Resultados adoptados por el GRECO ${ }^{1}$ en

1 El Grupo de Estados contra la Corrupción del Consejo de Europa, conocido por su acrónimo GRECO, se estableció a través de una decisión del Comité de Ministros del Consejo de Europa en mayo de 1998. Este Grupo tiene como objetivo la mejorar la capacidad de los Estados miembros que lo integran en la lucha contra la corrupción. Vid. Resolución (98) 7 del Comité de Ministros del Consejo de Europa, adoptada en su $102^{a}$ Sesión el 5 de mayo de 1998, autorizando el establecimiento del Grupo de Estados contra la Corrupción; y Resolución (99) 5, adoptada el 1 de mayo de 1999, estableciendo el Grupo de Estados contra la Corrupción (GRECO); Vid. en detalle sobre el funcionamiento del GRECO en el Estatuto de Funcionamiento del Grupo de Estados contra la Corrupción, que figura como anexo a la Resolución (99) 5, y el Reglamento interno del funcionamiento del GRECO (2012) 26E, adoptado por el Plenario de Representantes en octubre de 1999, modificado por última vez en octubre de 2012; puede consultarse una copia del mismo en la web del Consejo de Europa http://www.coe.int/t/dghl/monitoring/greco/documents/ 
julio de $2016^{2}$ y en diciembre de $2017^{3}$ en los que se evalúa y controla el grado de cumplimiento de las medidas y recomendaciones relativas a la prevención de la corrupción que el propio Grupo de Estados contra la Corrupción del Consejo de Europa dirigió a España en su pasado Informe de Evaluación de 2014 , y del que ya dimos cumplida cuenta en su día 5 .

Se trata, por tanto, del segundo y del tercero de los informes sobre España a través de los cuales se sustancia la Cuarta Ronda ${ }^{6}$ de evaluaciones del GRECO ${ }^{7}$, cuyo objetivo específico consiste en la prevención de la corrupción respecto de los miembros del Parlamento, los Jueces y los Fiscales ${ }^{8}$; si bien, tal y como hemos

Greco(2012)26_RulesOfProcedure_EN.pdf (consulta 30.12.2016). En adelante nos referiros a este documento como Estatuto del GRECO.

2 GRECO (CONSEJO DE EUROPA), Informe de Resultados-España: Prevención de la corrupción respecto de los miembros del Parlamento, Jueces y Fiscales. Cuarta Ronda de Evaluación, GRECO RC4 (2016)1, adoptado por el $72^{\circ}$ Pleno el 1 de julio de 2016 y publicado el 10 de octubre de 2016. Puede consultarse en inglés en la siguiente Web del GRECO: http://rm.coe.int/CoERMPublicCommonSearchServices/DisplayDCTMContent? documentId=09000016806ca04a (consulta 10.01.2017). El propio Informe recomienda su traducción al castellano por parte de las Autoridades españolas, si bien a fecha de cierre de este trabajo esta traducción no está aún disponible en la web del Consejo de Europa. En adelante nos referiremos a este informe como Informe 2016.

3 GRECO (CONSEJO DE EUROPA), Informe Provisional de Resultados-España: Prevención de la corrupción respecto de los miembros del Parlamento, Jueces y Fiscales. Cuarta Ronda de Evaluación, GRECO RC4 (2017)18, adoptado por el $78^{\circ}$ Pleno el 8 de diciembre de 2017 y publicado el 3 de enero de 2018. Puede consultarse en inglés en la siguiente Web del GRECO: https://rm.coe.int/fourth-evaluation-round-corruption-prevention-in-respect-of-members-of/1680779c4d (consulta 10 de enero de 2017). Al igual que en el anterior de 2016, también en este Informe se recomienda su traducción al castellano por parte de las Autoridades españolas, si bien a fecha de cierre de este trabajo esta traducción no está aún disponible en la web del Consejo de Europa. En adelante nos referiremos a este informe como Informe 2017.

4 GRECO (CONSEJO DE EUROPA), Informe de Evaluación-España: Prevención de la corrupción respecto de los miembros del Parlamento, Jueces y Fiscales. Cuarta Ronda de Evaluación, GRECO EVAL IV Rep (2013) 5E, adoptado por el $62^{\circ}$ Pleno el 6 de diciembre de 2013 y publicado el 15 de enero de 2014. Puede consultare una traducción al castellano en la siguiente Web del GRECO http://www.coe.int/t/dghl/monitoring/greco/ evaluations/round4/Eval\%20IV/GrecoEval4(2013)5_Spain_ES.pdf (consulta 27 diciembre de 2016). En adelante nos referiremos a él como Informe 2014.

5 Vid. CAMISÓN YAGÜE, J.A., «El informe sobre prevención de la corrupción en España del grupo de estados contra la corrupción del consejo de Europa (2014)», TRC, n ${ }^{\circ} 34,2014$, pp. 395-412. 8

6 Art. 10. 2. del Estatuto del GRECO «La evaluación se dividirá en rondas. Una ronda de evaluación es un periodo de tiempo determinado por el GRECO, durante el que se llevará a cabo un proceso de evaluación para evaluar el cumplimiento de los miembros con las disposiciones seleccionadas que se contienen en los Principios Rectores y otros instrumentos legales internacionales adoptados en cumplimiento del Programa de Acción contra la Corrupción». Téngase en cuenta que el GRECO usa parámetro de control los veinte Principios Rectores en la lucha contra la corrupción adoptados por el Comité de Ministros del Consejo de Europa en noviembre de 1997, así como otros instrumentos legales internacionales en la materia. Resolución (97) 24 del Comité de Ministros sobre los Veinte Principios Rectores para la lucha contra la corrupción, adoptada en su $101^{\circ}$ Sesión el 6 de noviembre de 1997.

7 Punto IV «Cuarta Ronda de Evaluación» del Informe Resumen del $62^{\circ}$ Pleno del Greco, celebrado en Estrasburgo del 2 al 6 de diciembre de 2013, GRECO 2013 18E, Puede consultare en inglés en la siguiente Web oficial del GRECO http://www.coe.int/t/dghl/monitoring/greco/documents/2013/ Greco(2013)18_Summary\%20Report\%2062_EN.pdf (consulta 30 de diciembre de 2016).

8 GRECO, Cuestionario sobre la Prevención de la corrupción respecto de los miembros del Parlamento, Jueces y Fiscales. Cuarta Ronda de Evaluación, GRECO (2012) 22E, aprobado por el $50^{\circ}$ Pleno del GRECO, 28 de 
indicado nos centraremos en este trabajo fundamentalmente en aquellas cuestiones referidas a Jueces y Magistrados.

Para ello hemos divido este estudio en dos partes. La primera de ellas tiene por objeto describir cómo se lleva a cabo el proceso de evaluación del GRECO, especialmente referido a la Cuarta Ronda, en la que se insertan los dos informes de seguimiento de 2016 y 2017, a fin de que el lector pueda efectivamente ubicarlos dentro del proceso de evaluación y conocer su naturaleza. Por otro lado, la segunda parte se centra en el análisis concreto de cada una de las específicas recomendaciones que el GRECO ha dirigido a España, para ello daremos cuenta de la recomendación recibida y de las subsiguientes actuaciones llevadas a cabo por las autoridades españolas para su aplicación, amén de la valoración recibida por los sucesivos informes de evaluación del GRECO. Finalmente, se aportarán unas breves conclusiones críticas, con las que se cierra este trabajo.

Es preciso antes de continuar agradecer aquí públicamente al GRECO por la información que nos ha sido facilitada para la realización de este artículo, así como la significativa colaboración prestada por la Dirección General de Cooperación Jurídica y Relaciones con la Confesiones del Ministerio de Justicia quien, mediante el Portal de la Transparencia, ha accedido a facilitarnos las copias de las contestaciones que el Estado español elevó en su día respecto de los sucesivos Informes del $\mathrm{GRECO}^{9}$, y a las que respectivamente nos referiremos a lo largo del texto como Informes de Situación de 2015 y de 2017.

\section{Sobre el proceso de evaluación de un Estado por parte del GRECO}

Antes de centrarnos tanto en el Informe de 2016 como en el Informe de 2017 del GRECO y sus respectivos contenidos, corresponde ahora ofrecer al lector una sucinta contextualización de ambos, a fin de que pueda situarlos correctamente dentro del alambicado y complejo proceso de evaluación que lleva a cabo

marzo-1 de abril de 2011, revisado por el $57^{\circ}$ Pleno del GRECO, 15 a 19 de octubre de 2012. Puede consultare en inglés en la siguiente Web oficial del GRECO http://www.coe.int/t/dghl/monitoring/greco/source/ Greco\%20(2012)\%2022E\%20Questionnaire\%20Eval\%20IV\%20REVISED.doc (consulta 10.07.2014). En concreto la Cuarta Ronda de Evaluaciones contemplaba entre sus objetivos el análisis de los mecanismos de prevención de la corrupción respecto de los Jueces y Magistrados en lo concerniente a los siguientes ámbitos concretos: la selección de Jueces y Magistrados, el desarrollo carrera judicial y la promoción, condiciones de trabajo, gestión de los procesos, principios éticos de la carrera judicial, normas de conducta, resolución de los conflictos de intereses, incompatibilidades, actividades complementarias permitidas, restricciones una vez abandonada la carrera judicial, casos y procedimientos de recusación y abstención, deberes de uso de la información relativa a los procesos, contactos con terceras partes, declaraciones de ingresos y patrimonio, supervisión y control del cumplimiento de los deberes éticos y de la carrera judicial y, finalmente, procesos de formación, concienciación y asesoramiento respecto de la corrupción.

9 Dirección General de Cooperación Jurídica y Relaciones con la Confesiones (Secretaria de Estado de Justicia) del Ministerio de Justicia: Situation Report whitin GRECO'S Fourth Evaluation Round, de 29 de septiembre de 2015 y GRECO’s Fourth Evaluation Round-Spain: Progress Report, de 27 de julio de 2017. 
este órgano encargado de la lucha contra la corrupción en el seno del Consejo de Europa.

En primer lugar, es preciso tener en cuenta que el GRECO realiza sus evaluaciones respecto de ámbitos determinados susceptibles de ser o de convertirse en focos de corrupción en los Estados mediante sucesivas «rondas». España actualmente se encuentra sometida a evaluación en el marco de la Cuarta Ronda, que arrancó en el año $2012^{10}$.

Una de las premisas fundamentales a tener en cuenta es que la evaluación de un Estado, dentro de cada una de las rondas que el GRECO acuerda, se realiza a través de varios informes sucesivos en los que se sustancian las dos fases principales en las que se organiza el proceso evaluador del GRECO: evaluación y control de cumplimiento.

La primera de ellas tiene por objeto valorar la situación del Estado y proponerle, en su caso, una serie de mejoras y recomendaciones; mientras que la segunda fase persigue, por su parte, verificar si el Estado finalmente cumple o no con las recomendaciones propuestas. Los dos informes sobre los que versa el presente trabajo correspondientes a 2016 y 2017 se incardinan dentro la segunda fase y son, por tanto, informes que evalúan el grado de cumplimiento alcanzado.

\subsection{Fase 1: El Informe de Evaluación}

La primera fase se sustancia en la realización de un primer informe al que se conoce como «Informe de Evaluación» y en él se examina a cada uno de los Estados respecto de los concretos y específicos aspectos a valorar en relación con la corrupción y su prevención, que han sido fijados específicamente para la ronda de que se trate.

En este Informe de Evaluación se efectúa un análisis de la situación existente en dicho Estado y, además, se incluyen en él las diversas medidas y recomendaciones ad hoc que el Estado debería adoptar para solucionar y prevenir los problemas relativos a la corrupción que hubieren sido detectados.

En el presente caso el Informe de Evaluación, tal y como ya anticipamos anteriormente, tiene fecha de 2014 y se enmarca dentro de la «Cuarta Ronda» de evaluación que se centró en el análisis de la Prevención de la corrupción respecto de los miembros del Parlamento, Jueces y Fiscales ${ }^{11}$.

10 La Primera Ronda de Evaluación, que tuvo lugar entre el 2000 y el 2002, se centró en las autoridades nacionales encargadas de la lucha contra la corrupción. Por su parte, la Segunda Ronda de Evaluación, celebrada entre 2003 y 2006, tuvo como objeto los resultados económicos de la corrupción, participación de las personas jurídicas en la corrupción blanqueo de capitales, y la corrupción en la Administración. La Tercer Ronda, que se lanzó en 2007, evaluó aquellos ámbitos concernientes a incriminaciones por corrupción y transparencia en la financiación de partidos políticos.

11 Vid. Supra. 


\subsection{Fase 2: Control del cumplimiento de las recomendaciones-Informe de Resultados}

Seguidamente tiene lugar la segunda fase, en la que se verifica el cumplimiento por parte del Estado evaluado de las recomendaciones contenidas en el «Informe de Evaluación». Dicha fase comienza con la obligación que tiene el Estado evaluado de remitir al GRECO un «Informe de Situación» en donde debe dar cuenta de las medidas llevadas a cabo para la puesta en marcha de las medidas y recomendaciones recibidas.

A la vista de este Informe de Situación, desde el GRECO se produce un segundo informe al que se denomina «Informe de Resultados» y cuyo objeto principal es la valoración del grado de cumplimiento e implementación de las recomendaciones. Este Informe de Resultados se centra particularmente en el análisis de la actividad del Estado evaluado respecto de cada una de las medidas propuestas el Informe de Evaluación.

Según establece el Reglamento Interno del GRECO, existen tres tipos de calificaciones para evaluar el grado de cumplimiento de cada una de las recomendaciones recibidas: la primera es que la medida «haya sido implementada de forma satisfactoria o bien que la misma se ha tratado de forma satisfactoria»; la segunda, «que la medida haya sido parcialmente implementada»; y la tercera «que la medida no haya sido implementada».

En función de las calificaciones recibidas, y siempre y cuando estas se correspondan bien con la segunda o la tercera, es decir, que el Estado no haya cumplido con las recomendaciones o solo lo hubiera hecho parcialmente, será necesario volver a llevar a cabo de nuevo el proceso de control del cumplimiento de las medidas recomendadas.

Para ello se requiere normalmente que el Estado evaluado aporte un segundo «Informe de Situación», que al igual que el anterior, dará lugar, en su caso, a la elaboración y aprobación de un nuevo «Informe de Resultados», para el que se seguirá el mismo procedimiento antes referido, y al que se le denomina en terminología del GRECO como «Informe de Resultados Provisional» (Interim Complince Report). Este Informe de Resultados Provisional tiene también, por lo tanto, como objeto analizar el grado de cumplimiento de las recomendaciones. Así, una vez que se aprueba el Informe de Resultados Provisional pueden pasar dos cosas. En primer lugar, que en ese segundo Informe se requiera al Estado para que aporte más información; lo que eventualmente da lugar la elaboración de un «Segundo Informe de Resultados Provisional» ${ }^{12}$. Y, en segundo lugar, puede suceder que, además, el GRECO considere «globalmente insatisfactorio» el grado de cumplimiento de las recomendaciones, con lo que a través de dicho Informe se insta a comenzar el «procedimiento de sanción»; que, como veremos

12 Como, por ejemplo, ha sucedido en esta Cuarta Ronda de Evaluación respecto de los Estados de Eslovenia o Letonia. 
seguidamente, se traduce en realidad en una suerte de medidas de presión internacional y un seguimiento especial de la implementación de las recomendaciones recibidas por el GRECO.

Finalmente, el proceso de evaluación, suele concluir con el llamado «Segundo Informe de Resultados», para el cual se sigue el mismo método de elaboración que para el Informe de Resultados. Dicho Segundo Informe aún no ha sido elaborado ni aprobado en el caso de España, por lo que a esta Cuarta Ronda del GRECO se refiere. En todo caso, llamamos la atención sobre el hecho de que su eventual adopción pueda tener lugar para principios de 2019, una vez que España haya procedido a informar de los avances realizados en la aplicación de las recomendaciones, para lo que tiene de plazo hasta finales de 2018.

Es importante señalar aquí, para clarificar la naturaleza de los Informes estudiados, que en el caso que nos ocupa, el de España, el Informe de 2016 se correspondería con el «Informe de Resultados» y el Informe de 2017 con el «Informe de Resultados Provisional». Por lo tanto, los Informes del GRECO de 2016 y 2017 que son objeto de este trabajo se incardinan dentro de esta fase de control del cumplimiento de las recomendaciones a las que nos hemos referido en el párrafo anterior, y se trata por tanto de dos informes cuya naturaleza es, tanto en uno como en otro caso, la de «Informes de Resultados»; si bien el segundo, que tiene la naturaleza de «Informe de Resultados Provisional», da lugar, como ya hemos dicho, a la activación de un control reforzado respecto de los incumplimientos, en tanto que el mismo constata expresamente que en España apenas se han seguido las recomendaciones recibidas ${ }^{13}$.

\subsection{Fase 3: "Control reforzado de incumplimientos»}

Así, si eventualmente se considera bien el Informe Provisional bien en el Segundo Informes de Resultados que el incumplimiento de las recomendaciones dadas a un Estado evaluado es «globalmente insatisfactorio» — como es el caso de España-, el GRECO puede aprobar que se ponga en marcha un proceso específico de control reforzado de incumplimientos.

Este consiste, fundamentalmente, en que dicho Estado debe presentar un nuevo informe o bien una serie de informes periódicos de situación sobre los progresos realizados en la puesta en marcha de las recomendaciones que en su día se contenía en el «Informe de Evaluación».

En su caso, y si los incumplimientos persisten, «a modo de sanción» el Presidente del GRECO podrá además dar cuenta mediante una carta de tal situación

13 Norma 31 del Reglamento Interno del GRECO, (2017) 13, que puede consultarse en https://rm. coe.int/rules-of-procedure-adopted-by-greco-at-its-1st-plenary-meeting-strasbo/168072bebd (consulta 3 de Febrero de 2017). 
a diversas autoridades del propio GRECO y del Consejo de Europa, llegando incluso a invitar a que dichas autoridades puedan dirigir a su vez también una carta a las autoridades nacionales, incluido al Ministro de Asuntos Exteriores del Estado en cuestión, llamando la atención sobre lo significativo del incumplimiento y la importancia de llevar a cabo acciones dirigidas a la implementación de las recomendaciones recibidas.

Dentro de este proceso específico de control reforzado sobre los incumplimientos puede también ocurrir que se requiera al Estado incumplidor para que se reciba en dicho Estado a una delegación de alto nivel, encabezada por el Presidente del Secretariado Ejecutivo del GRECO. El objeto de esta visita es hacer ver a ese Estado la significativa importancia de cumplir con las recomendaciones que se le han dirigido. Finalmente, el GRECO podrá también aprobar una declaración pública de incumplimiento, que acompañará de un informe de las actividades llevadas a cabo por el Estado en relación con las recomendaciones recibidas ${ }^{14}$.

\section{Sobre la elaboración de los Informes de Resultados de 2016 y 2017} referidos a España

Aunque ya nos hemos referido al proceso de evaluación, consideramos oportuno proceder ahora a dar cuenta pormenorizada de cómo se sustancia en concreto la elaboración y ulterior aprobación del Informe de Resultados; dado que tanto el 2016 como el 2017 lo son y suponen el objeto de nuestra investigación.

Así, los específicos trámites para la elaboración de estos Informes de Resultados, que se insertan dentro de la fase de control del cumplimiento antes referida, cuentan con tres pasos: elaboración del borrador del informe, revisión y, finalmente, aprobación.

\subsection{Paso 1: Elaboración del borrador del informe}

En primer lugar y por lo que respecta a la elaboración del Informe de 2016, debemos significar que para su confección se tuvieron en cuenta básicamente dos fuentes. De una parte, el Informe de Situación que las Autoridades Españolas (en concreto, la Dirección General de Cooperación Jurídica y Relaciones con la Confesiones del Ministerio de Justicia) remitieron al GRECO el 29 de septiembre de $2015^{15}$; en tanto en cuanto, tal y como indica el Reglamento interno

14 Norma 32 del Reglamento Interno del GRECO, (2017) 13. Téngase en cuenta que el GRECO ha aprobado una nueva norma 32 revisada que se aplicará en la Quinta Ronda de Evaluación y siguientes.

15 Téngase en cuenta que el GRECO amplió, por propia iniciativa, el plazo para la remisión del Informe hasta finales de septiembre de 2015, que originalmente concluía tres meses antes. 
del GRECO, debe ser el propio Estado, mediante el correspondiente «Informe de Situación» quien voluntariamente ha de dar cuenta de las medidas adoptadas en ejecución de las recomendaciones recibidas en el Informe de Evaluación ${ }^{16}$.

Y, de otra parte, se basa también en las apreciaciones realizadas por dos informadores del propio GRECO: el islandés Björ Thorvalsson, que se encargó de lo relativo a las y los miembros del Parlamento, y la italiana Laura Paesano, quien fue designada para aquello concerniente a las instituciones judiciales ${ }^{17}$. Estos dos informadores con la ayuda de la Secretaría del GRECO se encargaron de redactar un primer borrador del Informe de Resultados ${ }^{18}$.

Por su parte, en lo referido al Informe de 2017, también se elaboró igualmente en base a dos fuentes. De una parte, mediante el Informe de situación que las Autoridades Españolas, en concreto la Dirección General de Cooperación Jurídica y Relaciones con la Confesiones del Ministerio de Justicia, remitieron al GRECO el 27 de julio de 2017. De otra parte, mediante las aportaciones de los dos informadores del GRECO, que para este Informe de 2017 fueron el islandés Magnús Gunnarsson, que se encargó de las recomendaciones dadas al Parlamento, y la italiana Emma Rizzato, quien fue designada para examen de las recomendaciones relativas a las instituciones judiciales ${ }^{19}$.

\subsection{Paso 2: Revisión}

El segundo paso, al que hemos denominado de revisión, comienza con el envío al Estado miembro del primer borrador del Informe, para que éste pueda realizar comentarios y apreciaciones sobre el mismo dentro del plazo de catorce días desde su recepción. Seguidamente, y a la luz de las observaciones y aportaciones del Estado miembro, se procede de nuevo conjuntamente por la Secretaría del GRECO y por los dos informadores a la redacción de un segundo borrador revisado del Informe ${ }^{20}$.

16 Apartado 2 de la Norma 30 del Reglamento Interno del GRECO (consulta 3 de febrero de 2018). Punto 170 del Informe 2014 invitaba a las Autoridades Españolas a presentar un informe sobre las medidas adoptadas para ejecutar dichas recomendaciones antes del 30 de junio de 2015.

17 Téngase en cuenta que, según dispone el Apartado 1 de la Norma 31 Revisada del Reglamento Interno del GRECO (Aplicada desde la Tercera Ronda de Evaluación), el propio GRECO seleccionará a dos miembros responsables que serán los encargados de preparar un informe de resultados; esta selección se basa en criterios como la participación en la primera evaluación y la similitud de los sistemas jurídicos o la proximidad geográfica con el miembro evaluado. En este caso, Björ Thorvalsson es el Jefe de la Delegación Islandesa del GRECO y Fiscal en la Oficina Especial de la Fiscalía Islandesa; mientras que Laura Paesano es Magistrada, asignada a la Oficina del Gabinete del Ministerio de Justicia Italiano.

18 Apartado 1 de la Norma 31 Revisada del Reglamento Interno del GRECO.

19 Magnus Gunnarsonn es Subdirector de la Fiscalía islandesa; mientras que Emma Riazzato es una Magistrada Italiana.

20 Apartados 3 y 4 de la Norma 31 Revisada del Reglamento Interno del GRECO. 


\subsection{Paso 3: Aprobación}

Finalmente, tiene lugar el paso de aprobación que se sustancia en el Pleno del GRECO, previo examen y debate del Informe, y en donde el Jefe de Delegación nacional actuará como representante del Estado miembro sobre el que versa el Informe. Es necesario apuntar que el resto de miembros del GRECO reciben el segundo borrador del Informe, que es el que somete a discusión y aprobación, con una antelación de al menos catorce días antes de la celebración del Pleno. También cabe señalar que, llegado el caso, el GRECO puede, incluso, posponer la aprobación del informe si se considera necesario aportar más información ${ }^{21}$.

En el caso estudiado los Informes de Evaluación objeto de este trabajo fueron aprobados respectivamente por el Pleno del GRECO, el 1 de julio de 2016 y el 8 de diciembre de 2017.

\section{RECOMENDACIONES DEL GREGO PARA EVITAR LA CORRUPCIÓN EN EL PODER JUDICIAL: CONTROL DE SU CUMPLIMIENTO}

Tal y como hemos indicado en la introducción de este trabajo, hemos considerado oportuno no proceder a un simple análisis cronológico de los dos informes estudiados, sino tratar cada una de las recomendaciones específicamente referidas al Poder Judicial, y ver cuáles ha sido en su caso los avances producidos al respecto de cada una de ellas.

Como no podría ser de otra manera el punto de partida debe ser recordar aquí cuales eran básicamente dichas recomendaciones que el GRECO dirigió a España en 2014, y que, recordemos, ya fueron objeto de análisis en otro trabajo anterior $^{22}$. Todas ellas manifestaban la gran preocupación de GRECO por la amenaza existente en nuestro Estado de que el otros poderes del Estado, y fundamentalmente el poder político, pudieran inmiscuirse en el funcionamiento del Poder Judicial, haciendo peligrar su independencia.

Sucintamente enumeradas esas recomendaciones eran fundamentalmente las siguientes: revisar la forma de elección del Consejo General del Poder Judicial, revisar el método de designación de Jueces para las Altas Magistraturas, la adopción de un código deontológico para Jueces y Magistrados y, finalmente, elevar los plazos máximos de instrucción referidos a los procedimientos disciplinarios contra Jueces y Magistrados ${ }^{23}$.

21 Apartados 5 y 7 de la Norma 31 Revisada del Reglamento Interno del GRECO.

22 Vid. CAMISÓN YAGÜE, J.A., «op. cit.», pp. 395-412.

23 Punto VI. «Recomendaciones y Seguimiento» del Informe 2014. 
De todas ellas, adelantamos aquí, ninguna ha sido a ojos del GRECO completamente observada, lo que ha dado lugar a que se inste la puesta en marcha de los procedimientos de control reforzado de incumplimientos a los que anteriormente hicimos referencia, concretamente, se solicita a España a enviar un nuevo Informe de Situación antes de 31 de diciembre de 2018 y, de otra parte, se insta a las Autoridades del GRECO a remitir a España una carta en la que se signifique la gran importancia de llevar a cabo las acciones oportunas para cumplir con las recomendaciones formuladas.

\section{Revisión de la forma de elección del Consejo General del Poder Judicial}

En su Informe de 2014 el GRECO recomendaba a España encarecidamente que revisara el marco legislativo concerniente a la elección de las y los miembros integrantes del Consejo General de Poder Judicial en lo referente a la participación del Congreso y del Senado en su elección, además de señalar que era precisa una mayor transparencia en lo que al funcionamiento del CGJP se refiere ${ }^{24}$.

Por su parte el Estado español señaló en su Informe de Situación de 2015 en contestación al GRECO que la elección de las y los integrantes del C.G.P.J. tenía una suerte de limitaciones y prescripciones establecidas en la propia Constitución española ${ }^{25}$, que implican la participación del Congreso y del Senado en el proceso de elección (art. 122.3 CE). Además, señalaba España que ya se llevó a cabo una reforma en $2013^{26}$, que tenía cuatro pilares fundamentales: primero, que cualquier Juez o Magistrado pudiera optar como candidato a miembro del Consejo, lo que previsiblemente arrojaría como resultado una composición más plural e independiente; segundo, se establecieron unas nuevas mayorías reforzadas para la elección que, demandando el consenso entre partidos, impedirían el bloqueo político; tercero, se simplificaba la estructura y los costes del CGPJ y, en cuarto lugar, se dotaba al CGPJ de una serie de abogados permanentes que habrían de coadyuvar a su buen funcionamiento. También señalaba el Estado que se habían llevado aprobado ciertas reformas en la Ley del Poder Judicial en 2015, al objeto de facilitar y agilizar el funcionamiento de la Comisión Permanente del CGPJ

24 Según el art. 557 de la L.O.P.J., tras la reforma de 2013, mantiene la elección de los miembros del turno de Jueces por parte de las Cortes Generales: cada una de la Cámaras debe elegir a diez vocales (cuatro del turno de juristas de reconocido prestigio y seis del turno de Jueces, respectivamente) por una mayoría de tres quintos. En principio, cualquier Juez o Magistrado que esté en servicio activo podrá ser candidato, siempre y cuando tenga el aval de otros veinticinco jueces o bien venga avalado por una asociación judicial.

25 Vid. in extenso sobre la evolución de la regulación del Poder Judicial en las Constituciones de España, ALZAGA VILLAAMIL, O.; «El poder judicial en la Historia Constitucional Española», ALZAGA VILLAAMIL, O.; Sociedad democrática y Constitución (Estudios y cabos sueltos), Madrid, Marcial Pons, 2018.

26 L.O. 4/2013, de 28 de junio, de reforma del C.G.P.J., por la que se modifica la L.O. 6/1985, de 1 de julio, del Poder Judicial. 
(art. 601 de la LOPJ) ${ }^{27}$. Finalmente, señalaba España, en referencia a la mayor transparencia del funcionamiento del Consejo el hecho de que desde julio de 2014 se había puesto en marcha el portal de transparencia del Poder Judicial, donde no solo se daba cuenta de las actividades realizadas sino, entre otras informaciones, también de su presupuesto ${ }^{28}$.

Ante esta contestación el GRECO estimó en su Informe de 2016 que «no se había cumplido con la recomendación», y que, aunque ciertamente debía darse un plazo de tiempo para ver cómo funcionaba la reforma de 2013, era necesario buscar fórmulas, que, salvando las indicaciones dadas en la Constitución, permitieran eliminar cualquier influencia por parte de las autoridades políticas en la elección de las y los miembros del poder judicial. Pues además el GRECO había podido constatar la existencia de diversas encuestas y estudios de opinión en los que se evidenciaba la percepción mayoritaria de que la Justicia en España no era independiente ${ }^{29}$. En lo que atañe a la transparencia el GRECO daba la bienvenida a los esfuerzos manifestados, pero requería a España para que en futuros informes diera cuenta no solo de los cambios normativos producidos sino también de su funcionamiento real ${ }^{30}$.

Posteriormente España volvió a presentar en julio de 2017 su correspondiente Informe de Situación en el que, en primer lugar, se indicaba que en el país se habían producido varios meses inestabilidad política durante 2016 que habían conducido a la repetición de elecciones lo que provocaba que el Gobierno estuviera «en funciones», lo que, a su vez impedía la presentación de proposiciones de Ley a las Cámaras y por tanto era imposible que el Ejecutivo pudiera actuar como motor de los cambios sugeridos ${ }^{31}$. No obstante, también se hacía eco en el Informe del Pacto de Gobierno que el Partido Popular había acordado con Ciudadanos, entre cuyas medidas estaban algunas directamente relacionadas con las recomendaciones del GRECO tales como, por un lado, la modificación de la LOPJ para que al menos los doce vocales del CGPJ, que deben proceder de la carrera judicial, fueran efectivamente nombrados solo por Jueces y Magistrados; o, por otro lado, la eliminación de toda intervención de los Parlamentos y Asambleas de las Comunidades Autónomas en la propuesta de los magistrados de los Tribunales Superiores de Justicia de las Comunidades Autónomas ${ }^{32}$.

27 L.O. 7/2015, de 21 de Julio, por la que se modifica la L.O. 6/1985, de 1 de julio, del Poder Judicial

28 Se puede acceder a este portal la siguiente dirección web: http:/www.poderjudicial.es/cgpj/es/ Temas/Transparencia/ (consulta 3.02.2018).

29 Punto 31 del Informe de 2016. Según se indica en el Informe el 75\% de los Jueces y Magistrados que contestaron a la encuesta sobre el Poder Judicial manifestaron que la independencia del mismo se encontraba amenazada, y el $54 \%$ de los que dijeron conocer la reforma de 2013 , indicaron que la misma no propiciaba mayor independencia judicial.

30 Puntos 27 a 30 del Informe 2016.

31 Art. 21 de la Ley 50/1997 del Gobierno.

32 Punto 102 y Punto 142 del Documento «150 Compromisos para mejorar España» (Acuerdo de Gobierno entre el Partido Popular y Ciudadanos), puede consultarse una copia en la siguiente página web: https://www.okdiario.com/img/2016/08/28/150compromisos.pdf (consulta 3.02.2018). 
Destacaba también España en su Informe que el Congreso de los Diputados había aprobado en diciembre de 2016, a propuesta del Partido Socialista, una iniciativa no legislativa expresamente dirigida a dar impulso al cumplimiento de las recomendaciones dadas por el GRECO en su Informe de 2014, en las que se trataba expresamente la cuestión del método de elección de los miembros del $\mathrm{CGPJ}^{33}$.

Y a mayor abundamiento, destacaba el Informe la puesta en marcha de una Subcomisión dentro de la Comisión de Justicia del Congreso en la que comenzarían a debatirse, entre otras materias, sobre la composición del C.G.P.J. y el nombramiento de sus integrantes ${ }^{34}$. Al respecto básicamente se ha debatido sobre si los vocales del CGPJ que provienen de la carrera judicial deben ser elegidos por los propios Jueces y Magistrados o si, en su caso, debe producirse algún tipo de intervención por el Parlamento. Debe en este punto recordarse, tal y como hace el propio Informe presentado por España, que el Tribunal Constitucional en su Sentencia 108/86 apreció que no era, en principio, inconstitucional que el Parlamento eligiera y nombrara a las y los miembros del $\mathrm{CGPJ}^{35}$. Refiere, además, el Informe presentado por España que tanto la Asociación de «Jueces para la Democracia» como el «Foro Judicial Independiente» ha propuesto que los vocales del turno judicial sean elegidos por los Jueces y Magistrados, algo que según indica el Gobierno ya sucede en la práctica, aunque «indirectamente», pues los doce vocales del turno judicial son propuestos por los propios Jueces y Magistrados a las Cámaras, que, recordemos, son quien en última instancia los eligen de entre los presentados.

Finalmente, el Informe provisional de Resultados de 2017 del GRECO concluye a la vista de las observaciones presentadas por España que la recomendación

33 En su Punto 1.a) de la Proposición no de Ley sobre el cumplimiento de las recomendaciones del Informe del Grupo del Consejo de Europa de Estados contra la Corrupción, aprobada por la Comisión de Justicia del Congreso de los Diputados el 20 de diciembre de 2016, instaba a: «a) Modificar el procedimiento de designación parlamentaria de los Vocales del Consejo General del Poder Judicial conforme a los siguientes criterios: convocatoria pública de las vacantes; evaluación de la competencia e idoneidad de los candidatos por un Comité Asesor de composición profesional variable cuyos miembros serán designados por insaculación entre los propuestos por los Grupos Parlamentarios; publicidad de los informes de evaluación; sesiones de audiencia en las correspondientes comisiones parlamentarias; aprobación por la mayoría requerida por las Cámaras competentes. No podrán acudir a esta convocatoria pública quienes en los últimos dos años hayan sido cargos electos, miembros del Gobierno, de gobiernos autonómicos o altos cargos de las Administraciones Públicas. Publicado en BOCG (Congreso de los Diputados) nº 80 (Serie D) de 3 de enero de 2017.

34 La denominación oficial de esta subcomisión es «Subcomisión de estudios y definición de la Estrategia Nacional de Justicia» y su puesta en marcha fue aprobada por el Congreso de los Diputados el 2 de febrero de 2017. Toda la información relativa a esta subcomisión y a los debates y a las diversas comparecencia que han tenido lugar en su seno puede consultarse en la siguiente dirección web oficial del Congreso: http:// www.congreso.es/portal/page/portal/Congreso/Congreso/Organos/SubPon?_piref73_1339276_73_1339269 _1339269.next_page $=/ \mathrm{wc} /$ detalleInformComisiones\&subComi $=$ S\&idOrgano $=303201$ \&idLegislatura $=12$ (consulta 3.02.2018).

35 STC 108/1986, de 29 de julio. Vid. FF.JJ. 5 a 13 en los que se trata in extenso la participación del Parlamento en la elección de parte de los miembros del CGPJ. 
referida a la «eliminar la intervención de otros poderes», especialmente los políticos, en el nombramiento de las y los miembros del CGPJ solo se ha «cumplido parcialmente»; pues si bien se valora positivamente la puesta en marcha de una Subcomisión parlamentaria, que habrá de conducir a una reforma normativa, es pronto aún para poder saber si la misma concluirá de forma satisfactoria respecto del cumplimiento de la recomendación. Anima el Informe a España a continuar el proceso de revisión del nombramiento de los vocales del turno judicial para eliminar cualquier sombra de injerencia política en su nombramiento, de modo tal que este quede totalmente en manos de los propios Jueces y Magistrados. Además, también insta el GRECO a seguir avanzando en un funcionamiento del CGPJ que sea más colegiado, en donde sus decisiones se adopten fundamentalmente por mayorías cualificadas y en donde sus miembros tengan una dedicación completa $^{36}$.

\section{Revisión del método de designación de Jueces para las Altas Magistraturas}

El Informe del GRECO de 2014 recomendaba a España que revisara el método de designación de los Jueces y Magistrados que ocupan las Altas Magistraturas, a fin de que el proceso llevado al cabo por el CGPJ no tuviera naturaleza discrecional; y en este sentido invitaba a España a que regulara mediante una norma de rango legal dichos procesos.

En respuesta a esta recomendación el Estado español en su primer Informe de Situación de 2015 indicó que el proceso de elección de las Altas Magistraturas no era totalmente discrecional en tanto que el mismo se sujetaba a los principios de mérito y capacidad y, además, toda designación era motivada $\mathrm{y}^{37}$, en su caso, quedaba sometida al eventual control judicial. A ello había que sumar la puesta en marcha de información pública al respecto de estos procesos de elección en el portal web de transparencia del C.G.P.J.. También señalaba España que el proceso de elección se encontraba ya normativizado en el Reglamento 1/2010 de 25 de febrero que regula la provisión de plazas de nombramiento discrecional en los órganos judiciales ${ }^{38}$; si bien se afirmaba además por España que se estaba estudiando cómo mejorar dichos procesos de selección.

36 Puntos 34 a 37 del Informe 2017.

37 Art. 632 LOPJ: (...) «2. En los Plenos que decidan las propuestas de nombramiento se dejará constancia de la motivación del acuerdo, con expresión de las circunstancias de mérito y capacidad que justifican la elección de uno de los aspirantes con preferencia sobre los demás. 3. La motivación podrá hacerse por remisión, en lo coincidente, a la motivación de la propuesta de la Comisión Permanente.»

38 Publicado en BOE $n^{\circ} 56$ de 5 de marzo de 2010. Reformado en dos ocasiones: Acuerdo de 7 de marzo de 2013, del Pleno del CGPJ, por el que se modifica el Reglamento 1/2010, de 25 de febrero, que regula la provisión de plazas de nombramiento discrecional en los órganos judiciales y Acuerdo de 31 de marzo de 2016, del Pleno del CGPJ, por el que se modifica el Reglamento 1/2010, de 25 de febrero, que 
Por su parte el Informe de Resultados de 2016 del GRECO significaba que España no había cumplido con la recomendación dada respecto de la elección de las Altas Magistraturas, pues ni se había revisado el alto grado de discrecionalidad existente ni tampoco se había avanzado en modo alguno respecto a la recomendación relativa a la necesidad de regular estos procesos mediante una ley. A ello se suma, además, que, según diversos estudios de opinión a los que el GRECO había accedido, el sesenta y siete por ciento de los Jueces y Magistrados manifestaban serias dudas sobre el respeto de los principios de mérito y capacidad en el proceso de selección de las Altas Magistraturas ${ }^{39}$.

Seguidamente España, en su respuesta a las observaciones del GRECO presentadas mediante su Informe de Situación de 2017, argüía la existencia de ciertos avances en el cumplimiento de esta recomendación. En primer lugar, refería que el Acuerdo de Gobierno entre el Partido Popular y Ciudadanos se había hecho eco de la misma, en tanto que en dos de sus puntos se establecía la necesidad, por un lado, acordar un nuevo sistema de promoción de Jueces y Magistrados en el que efectivamente se tuviera en cuenta el mérito, la capacidad, la habilidad, la experiencia y la idoneidad para el desempeño del cargo y; por otro lado, la conveniencia de eliminar la posibilidad de participación de las Asambleas y Parlamentos autonómicos en la propuesta de candidatos y/o candidatas para los Tribunales Superiores de las Comunidades Autónomas ${ }^{40}$.

También indicaba España, por medio del Informe elaborado por el Gobierno, que en la Subcomisión creada en el seno de la Comisión de Justicia del Congreso (a la que ya nos referimos en el apartado anterior) se estaba efectivamente discutiendo esta cuestión. Así en este sentido se había tratado en su seno tanto la posibilidad de ampliar el número de años de la duración del nombramiento en las Altas Magistraturas, como también fórmulas para eliminar la participación de los respectivos Parlamentos de las Comunidades Autónomas en el nombramiento de las y los Magistrados del CGPJ. Además, en el Informe se señalaba que la Asociación Profesional de la Magistratura y el Foro Judicial Independiente habían propuesto específicamente que tanto la Presidencia de las Audiencias Provinciales como la de los Tribunales Superiores de Justicia fueran efectivamente elegidas por las los integrantes cada uno de estos respectivos órganos judiciales.

Finalmente, en su Informe de Resultados de 2017 el GRECO concluyó que España no había cumplido con la recomendación que le había sido dirigida pues no se constaba la existencia de ninguna reforma que limitara la discrecionalidad en el nombramiento de las Altas Magistraturas; pues es necesario que, tal y como

regula la provisión de plazas de nombramiento discrecional en los órganos judiciales, en lo relativo a los órganos de la jurisdicción militar.

39 Puntos 33 a 35 del Informe 2016.

40 Punto 141 y Punto 142 del Documento «150 Compromisos para mejorar España» (Acuerdo de Gobierno entre el Partido Popular y Ciudadanos), puede consultarse una copia en la siguiente página web: https://www.okdiario.com/img/2016/08/28/150compromisos.pdf (consulta 3.02.2018). 
se recomendaba, se fijen objetivos, criterios y procesos específicos y concretos para la selección de estas magistraturas de forma que efectivamente se garantice el mérito y la capacidad. Así, para concluir con este apartado, cabe señalar que el GRECO saluda y valora positivamente en su Informe que, al menos, se esté discutiendo en España sobre esta problemática respecto de los nombramientos de aquellos que han de ocupar la presidencia de las Audiencias Provinciales o los Tribunales Superiores de Justicia de las CCAA, pero sugiere que se incluya también en el debate a la Audiencia Nacional y al Tribunal Supremo ${ }^{41}$.

\section{Adopción de un código deontológico para Jueces y Magistrados}

La tercera de las recomendaciones más significativas que el GRECO había dirigido a España en su Informe de Evaluación de 2014 era la necesidad de que se adoptara un Código deontológico específico para Jueces y Magistrados en el que, entre otras materias, se contemplaran aquellas cuestiones específicamente referidas a los conflictos de intereses; así como, en su caso, el establecimiento de un específico órgano encargado de asesorar a las y los miembros de la carrera judicial en esta materia.

Así España, en su Informe de Situación de 2015, indicaba en relación con esta recomendación que desde el 7 de mayo de dicho año se había acordado la iniciativa de publicar los datos de patrimonio de las y los miembros integrantes de la Comisión Permanente del C.G.P.J. ${ }^{42}$. En este sentido se remarcaba también la reforma operada respecto del inclusión de un nuevo apartado 5 del artículo 579 de la LOPJ en donde específicamente se prescribía que tanto el Presidente, como los Vocales y el Secretario General del C.G.P.J. están sujetos al deber de efectuar una declaración de bienes y derechos y del control y gestión de activos financieros de los que sean titulares ${ }^{43}$. A ello añadía además España en su Informe el hecho de que el CGPJ adoptó en julio de 2014 un Convenio Marco de colaboración con Transparencia Internacional España al objeto de implementar una «verdadera política de transparencia» que diera efectivo cumplimiento a la Ley 19/2013 de Transparencia, acceso a la información y buen gobierno ${ }^{44}$. Finalmente se hacía

41 Puntos 34 a 37 del Informe 2017.

42 Estas declaraciones, referidas a las y los actuales miembros de la Comisión Permanente, pueden consultarse en la siguiente página web del portal de Transparencia del CGPJ: http://www.poderjudicial.es/cgpj/ es/Temas/Transparencia/Actividad-Economico-Financiera/Altos-Cargos--Retribuciones---indemnizaciones-y-declaraciones-de-bienes/ (consulta 3.02.2018).

43 Apartado introducido por la L.O. 7/2015, de 21 de Julio, por la que se modifica la L.O. 6/1985, de 1 de julio, del Poder Judicial. Esta declaración debe producirse según indica el apartado 5 del art. 579 LOPJ en los términos previstos en los arts. 17 y 18 de la Ley 3/2015, de 30 de marzo, reguladora del ejercicio del alto cargo de la Administración General del Estado, con las adaptaciones que sean precisas a la organización del CGPJ, y que se recogerán específicamente el Reglamento de Organización y Funcionamiento del mismo.

44 Este Convenio Marco, prorrogado en 2016 hasta julio de 2018, puede consultarse en la siguiente página web del portal de Transparencia del CGPJ: http://www.poderjudicial.es/cgpj/es/Temas/Transparencia/ Buen-Gobierno-y-Codigo-etico/Convenio-CGPJ---Transparencia-Internacional-Espana/ (consulta 3.02.2018). 
también referencia a la puesta en marcha desde 2014 por parte del CGJP de un Grupo de Trabajo encargado de la elaboración de un Código deontológico ${ }^{45}$. Finalmente, en lo que la específica recomendación del establecimiento de un órgano asesor sobre conflictos de intereses para jueces y fiscales, se respondía por parte de España que tales funciones ya eran desempeñadas por los servicios internos del CGPJ.

Seguidamente el GRECO en su Informe de Resultados de 2016 ya indicaba que España solo estaba cumpliendo parcialmente con la recomendación dada; y constataba que el Estado español se había adherido al Código Iberoamericano de Ética Judicial mediante decisión del C.G.P.J. ${ }^{46}$. Además se felicitaba del establecimiento de un Grupo de Trabajo específicamente establecido para la elaboración del Código Ético y de su composición multidisciplinar, pues además de miembros de la carrera judicial se integraban en él también a expertos académicos en cuestiones de ética y de sociología ${ }^{47}$; algo que habría de dar lugar a un interesante proceso de reflexión interna y de toma de conciencia respecto de esta cuestión no solo por los integrantes del Poder Judicial sino también por la propia sociedad española. No obstante, el GRECO no se daba completamente por satisfecho pues, aunque sí se estaba en el buen camino, era preciso ver y comprobar cuál era el resultado final de tales trabajos. Por otra parte, también tomaba nota el GRECO de que los Servicios internos del CGPJ y, específicamente, su Sección del Departamento de Personal asesoraba a Jueces y Magistrados sobre los posibles conflictos de interés ${ }^{48}$.

En su Informe de Situación de 2017 España daba cuenta al GRECO de la aprobación en diciembre de 2016 por unanimidad del CGPJ del Código deontológico, bajo la denominación de «Principios de Ética Judicial» ${ }^{49}$, en los cuales se

45 Acuerdo IV - 1. De Constitución de un Grupo de Trabajo que impulse la elaboración de un Código Ético de la Carrera Judicial, adoptado por la Comisión Permanente del CGPJ en su reunión de 9 de abril de 2014. Dicho grupo estaba presidido por el Presidente del CGPJ y del TS, C. Lesmes y coordinado por los Vocales R. Bach y F. Grande-Marlaska, formando parte del mismo: J.A. Xiol, Magistrado del TC, C. Gómez, Presidente de la AP de Baleares, P. Llarena, Presidente de la AP de Barcelona; J.J. Toharia, Catedrático de Sociología; A.Cortina, Catedrática de Ética, y M. Olmedo, Letrado del CGPJ.

46 Acuerdo del Pleno del CGPJ de 25 de febrero de 2016 por que se acordó «ratificar, en tanto en cuanto no se apruebe un código ético propio para la carrera judicial española, los principios que inspiran el Código Ético Iberoamericano de Ética Judicial, adoptado en la Cumbre Judicial Iberoamericana de 2006 y modificado en 2014». Puede consultarse un copia del mismo en la web de la Red Iberoamericana de escuelas judiciales: http://www.riaej.com/ system/files_force/CIEJ_reformado_2014.pdf?download=1 (consulta 3.02.2018).

Se hacía especial mención en dicho acuerdo al hecho de que el código que «cumplía con los principios esenciales en materia de deontología judicial proclamados por la Red Europea de Consejos de Justicia». Puede consultarse en inglés las distintas Opiniones formuladas por esta Red en la siguiente página web: https://www.encj.eu/ index.php?option $=$ com_content \&view $=$ category\&layout $=$ blog\&id $=24 \&$ Itemid $=98 \&$ lang $=$ en En concreto vid. in extenso Declaración de Londres sobre la deontología de los jueces (2010), promovida por la Red Europea de Consejos de Justicia. (consulta 3.02.2018).

47 Vid. supra nota 45.

48 Puntos 37 a 41 del Informe 2016.

49 Documento aprobado por el Pleno del CGPJ en su sesión del día 20 de diciembre de 2016, en bases al texto acordado que fuera aprobado en la sesión celebrada el día 16 de diciembre de 2016 por el grupo de trabajo sobre ética judicial. Puede consultarse en la siguiente página web: http://www.poderjudicial.es/stfls/ 
contemplan no solo las reglas, principios y valores de independencia, imparcialidad, integridad, cortesía, diligencia y transparencia que han de observarse en el ejercicio de la actividad judicial; sino también el establecimiento de una específica Comisión de Ética Judicial que tiene por misiones: «emitir dictámenes por escrito sobre las consultas relativas a casos concretos que le hagan las Salas de Gobierno de los Tribunales, las Juntas de Jueces, las Asociaciones Judiciales o cualquier juez o jueza en servicio activo»; así como "promover la difusión y el conocimiento de los principios y proposiciones de ética judicial», amén de "contribuir al desarrollo de las funciones atribuidas al C.G.P.J. en la coordinación y la colaboración con otras comisiones judiciales de ética»; y, finalmente y de manera excepcional, también corresponde a esta Comisión de Ética «elaborar informes sobre asuntos o cuestiones que resulten de interés general y estén relacionados con el comportamiento ético que con arreglo a los principios recogidos en este texto se espera de los jueces y juezas, a instancia de hagan las Salas de Gobierno de los Tribunales, las Juntas de Jueces o las Asociaciones Judiciales». Esta comisión está compuesta por siete miembros: un juez, tres magistrados, dos magistrados del Tribunal Supremo y un Profesor de reconocido prestigio bien de Ética, bien de Filosofía del Derecho o bien de Filosofía Moral ${ }^{50}$.

Finalmente, el GRECO en su Informe provisional de Resultados de 2017 daba la bienvenida a la aprobación del Código deontológico especialmente referido a jueces y fiscales. Sin embargo, indicaba que la recomendación dada originalmente a este respecto solo se había cumplido parcialmente, pues se hace necesario esperar a conocer cómo se aplicaba en la práctica dicho Código, así como verificar la efectiva puesta en marcha de la Comisión de Ética y la elección de sus miembros; para lo cual recordaba que es imprescindible la colaboración del CGPJ y la correspondiente dotación de los medios necesarios ${ }^{51}$.

\section{Ampliación de los plazos de instrucción referidos a los procedimientos disciplinarios contra Jueces y Magistrados}

La cuarta y última recomendación significativa que el GRECO dirigió a España en su Informe de Evaluación de 2014 fue la revisión de los plazos máximos de instrucción de los procedimientos disciplinarios seguidos contra Jueces y Magistrados, pues su fijación en seis meses era manifiestamente disfuncional para su efectiva conclusión.

España contestó en su Informe de Situación de 2015 que la fijación de dicho plazo máximo de seis meses obedecía a lo regulado la Ley Orgánica del Poder Judicial en donde se fijaba tal plazo con carácter general para todos los procedimientos

CGPJ/TRANSPARENCIA/FICHEROS/20161221\%20Principios\%20de\%20Ética\%20Judicial\%20-\%20 Pleno\%20CGPJ\%2020.12.2016.pdf (consulta 3.02.2018).

50 Parte II «La Comisión de Ética Judicial» de los Principios de Ética Judicial.

51 Puntos 44 a 48 del Informe 2017. 
disciplinarios, salvo que razones excepcionales se pudiera demandarse un mayor plazo $^{52}$.

Como no podía ser de otra manera ante tal contestación dada por España, el GRECO constató en su Informe de Resultados de 2016 que España no había cumplido con la recomendación dada sobre la ampliación del plazo y que, además, se había detectado un significativo aumento de las Sentencias del Tribunal Supremo en la que se revocaban las sanciones disciplinarias contra Jueces y Magistrados adoptadas por el CGPJ por no respetarse los plazos del procedimiento ${ }^{53}$. A mayor abundamiento el GRECO significaba que el plazo máximo de duración tanto para los procesos disciplinarios de los funcionarios judiciales y como para los Secretarios Judiciales («Letrados de la Administración de Justicia») se fija en doce meses, lo que supone una evidente y significativa diferencia ${ }^{54}$.

En su Informe de Situación de 2017 España no hizo ninguna referencia a esta recomendación, salvedad hecha de que, tal y como luego señalaba el propio GRECO, la Subcomisión de estudios y definición de la Estrategia Nacional de Justicia, que se había constituido en el seno de la Comisión de Justicia del Congreso, podría ocuparse de esta cuestión.

Finalmente, el GRECO en su Informe provisional de Resultados de 2017 se reafirmaba, por un lado, en su constatación de que España nada había hecho en referencia a esta recomendación y, por otro lado, en el hecho de que el mantenimiento del plazo máximo de seis meses para la instrucción de expedientes disciplinarios a Jueces y Magistrados no solo suponía un agravio comparativo con los doce meses fijados para los funcionarios y Secretarios Judiciales; sino que también seguían aumentado el número de anulaciones del Tribunal Supremo respecto de sanciones impuestas por el CGJP en base a que en el procedimiento sancionador se había superado el tiempo máximo de instrucción ${ }^{55}$.

\section{CONCLUSIONES}

Cuatro años han pasado ya desde que el GRECO recomendara encarecidamente a España en 2014 una serie de medidas directamente orientadas tanto a garantizar la independencia del Poder Judicial como a mejorar su funcionamiento;

52 Apartado 5 del art. 425 LOPJ: «La duración del procedimiento sancionador no excederá de seis meses. Cuando, por razones excepcionales, se prolongase por mayor plazo, el instructor delegado deberá dar cuenta cada diez días del estado de su tramitación y de las circunstancias que impiden su conclusión a la autoridad que bubiere mandado proceder».

53 Puntos 43 a 45 del Informe 2016.

54 Vid. por ejemplo Art. 185 del RD 1608/2005, de 30 de diciembre, por el que se aprueba el Reglamento Orgánico del Cuerpo de Secretarios Judiciales. Duración del procedimiento disciplinario: «1) La duración del procedimiento disciplinario no excederá de doce meses. Vencido este plazo sin que se haya dictado y notificado la resolución que ponga fin al procedimiento, se producirá su caducidad (...)».

55 Puntos 50 a 53 del Informe 2017. 
a ello se suman, como hemos visto, dos sucesivos Informe de Resultados de 2016 y 2017 en los que, evaluada la acción de Estado al respecto, se constata que ninguna de dichas recomendaciones ha sido efectivamente cumplida en su totalidad.

Por todo ello, la primera conclusión de este trabajo no puede ser otra que afirmar que una oscura sombra sigue cerniéndose nuestra Justicia; en tanto en cuanto su independencia, que, no olvidemos, es uno de los pilares fundamentales de su legitimación, continúa seriamente amenazada en un momento de nuestra historia en el que está llamada a resolver importantes asuntos de significativa trascendencia para el Estado, tales como los casos de corrupción política, que todos conocemos; así como, también, a adoptar importantes decisiones respecto de las responsabilidades penales que, en su caso, puedan derivarse de proceso independentista catalán.

En segundo lugar, cabe también llamar la atención sobre la conveniencia de que el propio Estado admita sin ambages que el grado de cumplimiento de las recomendaciones del GRECO es manifiestamente mejorable, pues en ocasiones las respuestas contenidas en los Informes de Situación que España eleva, tal y como hemos visto, son, cuanto menos, calificables como un vano intento por justificar aquello que no tiene justificación posible, esto es, que España sigue incumpliendo con dichas recomendaciones. Así sucede, por ejemplo, respecto de la duración de los procedimientos disciplinarios contra Jueces y Magistrados, donde los datos y evidencias dadas por el GRECO son ciertamente demoledoras, mientras que las justificaciones dadas por España rallan, por su parte, en lo esperpéntico. También, en aquello que tiene que ver con la despolitización de la elección de los miembros del CGPJ o en la elección de las Altas Magistraturas, queda constatada la falta de avances significativos que, por otra parte, aún habrán de hacerse esperar pues, como se sabe, el clima político actual no parece propicio para los grandes consensos que estas cuestiones de la Justicia necesitan y demandan.

Finalmente, es preciso decir que, al menos, una pequeña esperanza de cambio se otea en el horizonte con la aprobación por parte del CGPJ de sus «Principios Éticos», cuya correcta eficacia y efectiva aplicación, tal y como indica el GRECO, quedan pendientes ser verificadas en el futuro.

$$
* * *
$$

TITLE: 2016 and 2017 Compliance Reports on the recommendations proposed by GRECO for the prevention of judicial corruption in Spain: Chronicle of non-compliances

ABSTRACT: This paper analyses the 2016 and 2017 Compliance Reports of Group of States against Corruption of Council of Europe (GRECO) about corruption prevention in Spain and it is focused on the study of its judiciary aspects. These Reports warn about the dangers of corruption in the Organs of Judiciary Government and in the election of Judges for High Courts caused by the political interference and also indicate that in Spain is not accomplished at all with the recommendations that GRECO addressed to the State in 2014. 
RESUMEN: Este trabajo analiza los Informes de Cumplimiento de 2016 y 2017 del Grupo de Estados contra la Corrupción del Consejo de Europa (GRECO) sobre prevención de la corrupción en España y se centra en el estudio de sus aspectos judiciales. Estos informes alertan sobre los serios peligros, provocados por la injerencia política, que acechan a los Organos del Poder Judicial y se proyectan en la elección de los Jueces de los Altos Tribunales. Finalmente, estos informes también indican que en España no se cumplen con las recomendaciones que GRECO dirigió al Estado en 2014 a este respecto.

KEY WORDS: Group of States against Corruption of Council of Europe, GRECO, Compliance Report, corruption, division of powers, Judiciary Power, Spain.

Palabras Clave: Grupo de Estados contra la Corrupción del Consejo de Europa, GRECO, Informe de Resultado, corrupción, división de poderes, Poder Judicial, España.

FECHA DE RECEPCIÓN: 10.10.2017

FECHA DE ACEPTACIÓN: 01.02.2018 